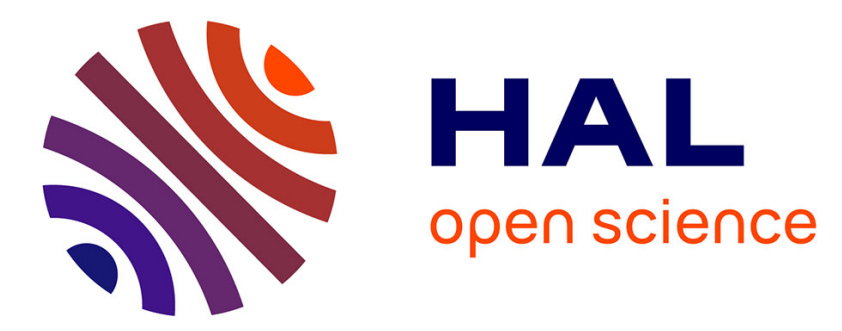

\title{
Multiple two-step oscillation regimes produced by the alto saxophone
}

Tom Colinot, Philippe Guillemain, Christophe Vergez, Jean-Baptiste Doc, Patrick Sanchez

\section{- To cite this version:}

Tom Colinot, Philippe Guillemain, Christophe Vergez, Jean-Baptiste Doc, Patrick Sanchez. Multiple two-step oscillation regimes produced by the alto saxophone. Journal of the Acoustical Society of America, 2020, 147 (4), pp.2406-2413. 10.1121/10.0001109 . hal-02549931

\section{HAL Id: hal-02549931 \\ https://hal.science/hal-02549931}

Submitted on 21 Apr 2020

HAL is a multi-disciplinary open access archive for the deposit and dissemination of scientific research documents, whether they are published or not. The documents may come from teaching and research institutions in France or abroad, or from public or private research centers.
L'archive ouverte pluridisciplinaire HAL, est destinée au dépôt et à la diffusion de documents scientifiques de niveau recherche, publiés ou non, émanant des établissements d'enseignement et de recherche français ou étrangers, des laboratoires publics ou privés. 
Multiple two-step oscillation regimes produced by the alto saxophone

Tom Colinot, ${ }^{1, \text { a) }}$ Philippe Guillemain, ${ }^{1}$ Christophe Vergez, ${ }^{1}$ Jean-Baptiste Doc, ${ }^{2}$ and Patrick Sanchez ${ }^{1}$

${ }^{1}$ Aix Marseille Univ, CNRS, Centrale Marseille, LMA, Marseille, France

${ }^{2}$ Laboratoire de Mécanique des Structures et des Systèmes couplés, Conservatoire National

des Arts et Métiers, Paris, France

(Dated: 27 March 2020) 
A saxophone mouthpiece fitted with sensors is used to observe the oscillation of a saxophone reed, as well as the internal acoustic pressure, allowing to identifiy qualitatively different oscillating regimes. In addition to the standard two-step regime, where the reed channel successively opens and closes once during an oscillation cycle, the experimental results show regimes featuring two closures of the reed channel per cycle, as well as inverted regimes, where the reed closure episode is longer than the open episode. These regimes are well-known on bowed string instruments and some were already described on the Uillean pipes. A simple saxophone model using a measured input impedance is studied with the harmonic balance method, and is shown to reproduce the same two-step regimes. The experiment shows qualitative agreement with the simulation: in both cases, the various regimes appear in the same order as the blowing pressure is increased. Similar results are obtained with other values of the reed opening control parameter, as well as another fingering.

a) colinot@lma.cnrs-mrs.fr 


\section{INTRODUCTION}

Various oscillating regimes, defined as the pattern of oscillations both mechanical and acoustical that correspond to the production of a periodic sound, have been observed and classified on bowed string instruments(Schelleng, 1973). The strongly non-linear friction law between bow and string leads to an oscillation pattern known as stick-slip motion, where the string sticks to the bow for a part of the period and then slips for another part of the period. The stick-slip phases may occur twice per period, leading to the so-called "double stick-slip" motion.

Reed conical instruments have often been compared to bowed strings, by virtue of the cylindrical saxophone approximation, which replaces the conical resonator with two parallel cylinders (Ollivier et al., 2004) because their impedance is similar in low frequency. In reed instruments, the analogous motion to stick-slip is called two-step motion (Ollivier et al., 2005). It consists in a beating reed regime, where the reed channel is closed for part of the period, and open for the rest of the period. The most common case, where the reed closure episode is shorter than half the period, is called standard two-step motion. Otherwise, the regime is called inverted. Standard and inverted two-step motions have been observed experimentally on a saxophone and predicted analytically on a cylindrical equivalent (Dalmont et al., 2000). Oscillating regimes showing more than one closure of the reed per period were never studied on the saxophone to our knowledge. They have been observed on a double reed instrument, the Irish Uillean pipes (Dalmont and Le Vey, 2014). To observe the signals produced by a wind instrument in playing situation, with a 
musician, an instrumented mouthpiece fitted with a reed displacement and pressure sensors can be used. Instrumented mouthpieces can help explain features of the produced sound, for instance spectral content on a saxophone (Guillemain et al., 2010) or transient descriptors on a clarinet (Pàmies-Vilà et al., 2018). They also provide a means to estimate some of the parameters of a physical model based on the dynamical behavior of the system (Muñoz Arancón et al., 2016).

This paper reports experiments in playing conditions exhibiting classic standard and inverted regimes, as well as double two-step motions, where the reed channel closes twice per period. To complete the study, we show that a simple saxophone model based on the input impedance of the saxophone used for the experiment is able to reproduce these double two-step regimes. The Harmonic Balance Method associated with continuation (Asymptotic Numerical Method) is used to obtain periodic signals corresponding to several control parameter combinations. The numerical simulations, in addition to experimental data, provide insights about the possible ways of transition between single and double two-step regimes, as well as the second register of the instrument. We also show that similar behavior occurs for neighboring fingerings and control parameter values. Describing and categorizing the oscillation regimes of the saxophone, as well as the musician's actions needed to obtain them, is among the first steps towards objective characterization of the ease of playing of an instrument. 


\section{EXPERIMENTAL OBSERVATION OF DOUBLE TWO-STEP MOTIONS ON}

\section{A SAXOPHONE}

\section{A. Experimental apparatus}

An instrumented mouthpiece is used to monitor the blowing pressure, the pressure inside the mouthpiece, and the position of the reed. It is shown in figure 1. It consists in a modified saxophone mouthpiece (Buffet-Crampon) incorporating two pressure probes: one going into the mouth of the musician and one into the mouthpiece, as well as an optical sensor (Everlight ITR8307) measuring the displacement of the reed. The pressure probe tubes are connected to a Honeywell TSCDRRN005PDUCV pressure sensor. The tubes have a radius of $0.55 \mathrm{~mm}$ and a length of $20 \mathrm{~mm}$ (mouth pressure) and $62 \mathrm{~mm}$ (pressure in the mouthpiece). According to (Guillemain et al., 2010), the transfer function of these capillary tubes is well represented by a model with non-isothermal boundary conditions (Keefe, 1984). An inverse filtering was performed on the pressure signals to compensate the effect of the probe tubes. Signals are then acquired using an NI USB-9234 card by National Instruments at a $51.2 \mathrm{kHz}$ sampling rate. Experimental signals displayed hereafter are not scaled or converted as this work focuses on qualitative study of the regime types. The instrumented mouthpiece is equipped with a saxophone reed (Rico Royal strength 2) and mounted on a commercial alto saxophone (Buffet-Crampon Senzo).

Throughout the remainder of the paper, a low $B$ fingering (written pitch) is studied. In concert pitch, the fundamental note expected with this fingering is a $D 3$ at the frequency 146.83 Hz. The input impedance of the saxophone for this fingering has been measured 


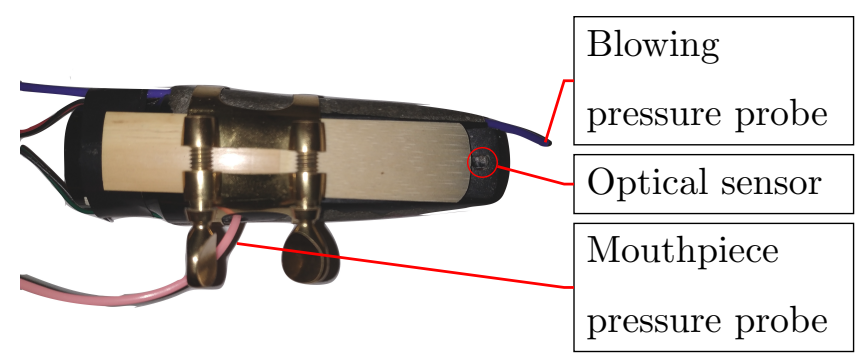

FIG. 1. Instrumented alto saxophone mouthpiece including pressure probes for the pressure in the mouth of the musician and in the mouthpiece, and an optical sensor measuring the displacement of the reed. The reed is pulled back so that the optical sensor is uncovered.

using the CTTM impedance sensor (Dalmont and Le Roux, 2008). Its modulus is displayed in figure 2. The B fingering, which produces the second lowest note on the instrument, is chosen because the double two-step regimes studied in this work tend to appear more easily on the lowest notes of the saxophone. Note that for this fingering, the note most commonly expected by musicians is the first register, whose frequency is around the first impedance peak. On this fingering, the first register is often hard to produce, especially for beginner musicians. This can be understood when looking at the impedance modulus curve on figure 2 , where the first peak is lower than the next three peaks: the upper resonances of the bore play a large part in the sound production, leading to a complicated sound production behavior. This profile of amplitude of the first few impedance peaks is also found in soprano and tenor saxophone (Chen et al., 2009). The lowest fingering $(B b)$ was not chosen, although it was tested, because it is more subject to producing undesired multiphonics and quasi-periodic regimes. 


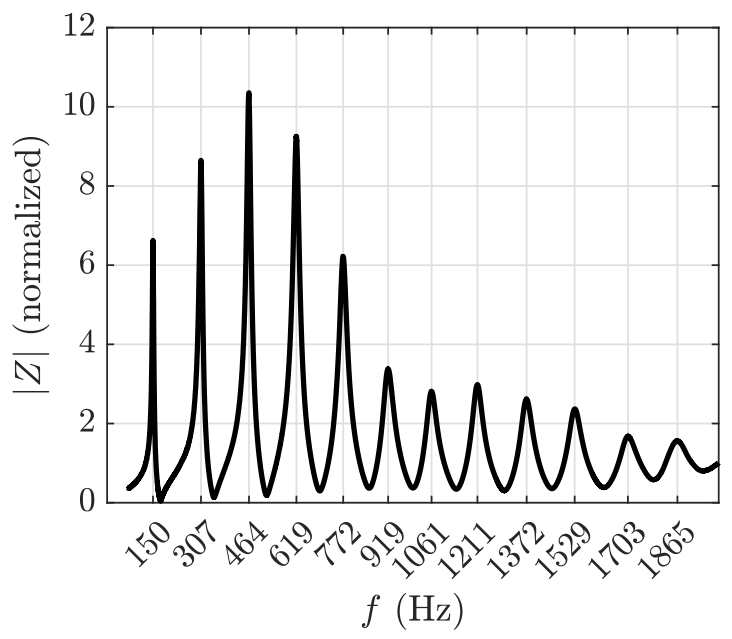

FIG. 2. Input impedance modulus measured for the studied fingering of the alto saxophone: low $B$ in written pitch. The modulus of the impedance is normalized by the characteristic impedance at the input of the instrument.

\section{B. Observation of single and double two-step oscillating regimes}

The main oscillating regimes of a saxophone are beating, which means that the reed channel closes completely during part of the cycle. They can be thought of as two-step motions (Ollivier et al., 2004) and classified as standard or inverted, depending on the relative duration of the open and closed episode. Different regimes can be obtained for the same fingering, just by varying the control parameters such as the blowing pressure. Figure 3 shows measured examples of these two-step regimes. The reed displacement signal was post-processed by substracting its moving average over a period, to be centered around 0 . The standard regime is characterized by an open episode and a short closed episode. As can be seen on figure 3 (a), the reed is opened - and displays small amplitude oscillations around the highest values of $x$ - for about $6 \mathrm{~ms}$. Its closure corresponds to the main dip in 
the waveform and it lasts for about $1 \mathrm{~ms}$ per period. For the inverted motion on figure 3 (b), the duration ratio is reversed: the reed channel is almost at its narrowest about $6 \mathrm{~ms}$ and opens wide briefly for about $1 \mathrm{~ms}$. Note that the standard regime is obtained for lower values of the blowing pressure than the inverted regime.

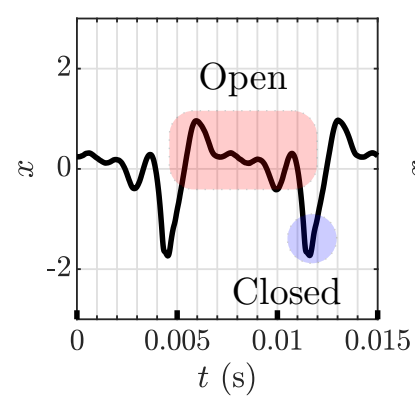

(a)

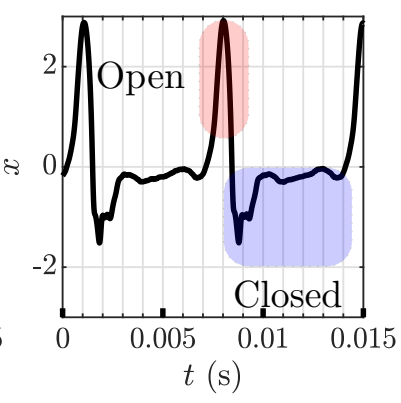

(b)

FIG. 3. Measured reed position for simple two-step motions: standard (a) and inverted (b). The reed channel is closed when $x$ is low. These waveforms correspond to different blowing pressures (see circle markers on figure 5).

The analogy with bowed string instruments suggests the apparition of other types of regimes. For example, under given excitation condition, bowed strings are subject to the double stick-slip phenomenon (Woodhouse, 2014), an oscillation regime where the string slips under the bow twice per period (instead of once for the standard Helmholtz motion). When transposed to conical reed instruments, this phenomenon corresponds to two closures of the reed channel per period. These regimes are observed experimentally on the low fingerings of the saxophone and they can be standard or inverted, as shown in figure 4. This oscillating regime can be called "double two-step". Note that the double two-step regime is distinct from second register regimes: it is a first register regime, as it produces the same note as 
the standard two-step regime. For the standard version of the double two-step regime, the closure episodes are about $1 \mathrm{~ms}$, almost the same duration as in the single standard two-step motion (figure 3, (a)). For the inverted double two-step regime, the short openings of the reed channel also last for about $1 \mathrm{~ms}$. For illustration purposes, the audible sound outside the instrument was recorded and short clips are provided as multimedia files 3, 4, 1 and 2 . Note that the audible sound corresponding to these double two-step regimes (Mm. 3 and 4 ) is clearly different from single regimes (Mm. 1 and 2). The difference in audible sound is less clear between a standard regime and its inverted counterpart.

Mm. 1. Sound recorded outside the resonator for the standard two-step motion, corresponding to the measured displacement shown in figure 3, (a).

Mm. 2. Sound recorded outside the resonator for the inverted two-step motion, corresponding to the measured displacement shown in figure 3, (b).

Mm. 3. Sound recorded outside the resonator for the double two-step motion, corresponding to the measured displacement shown in figure 4, (a).

Mm. 4. Sound recorded outside the resonator for the inverted double two-step motion, corresponding to the measured displacement shown in figure 4, (b).

In order to estimate the relative regions of production of each kind of regime in the control parameter space, a blowing pressure ramp is performed by a musician and recorded using an instrumented mouthpiece for the B fingering of the test saxophone. The musician 


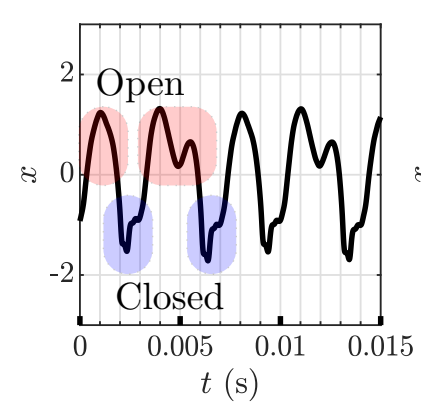

(a)

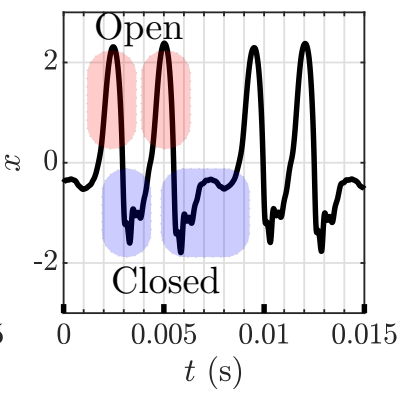

(b)

FIG. 4. Measured reed position for double two-step motions: standard (a) and inverted (b). These waveforms correspond to different blowing pressures (see circle markers on figure 5).

sees the evolution of the blowing pressure parameter in real-time on a screen. The player makes as little embouchure adjustments as possible and focuses on increasing the blowing pressure progressively. Results are shown in figure 5. This ramp was obtained in a single breath after several tries. For clarity, the blowing pressure signal is smoothed by a moving average with a rectangular window, adjusted to reject the fundamental frequency of the oscillations and keep only the slowly varying value of the signal. Regimes are classified automatically based on the ratio of duration of the open and closed reed episodes. The reed displacement signal is high-pass filtered in order to remove the DC component. The reed is then considered "open" when the displacement signal is above 0 and "closed" when it is below 0 . The ratio between closed duration and oscillation period is then computed and averaged over 4 periods. Thresholds are defined arbitrarily to separate between the different types of regimes, at $0.1,0.25,0.5,0.6$ and 0.8 (see dotted lines on figure 5). Looking at the pressure ramp in its entirety shows a possible order of the regimes when increasing the blowing pressure: standard and double two-step motions, second register, and inverted 
double then inverted two-step motions. Note that in this ramp, the episode between 1 and 2 seconds with a closure ratio of little above 0.25 is actually a quasi-periodic oscillation, with the actual double two-step oscillation starting at around 2.3 seconds.

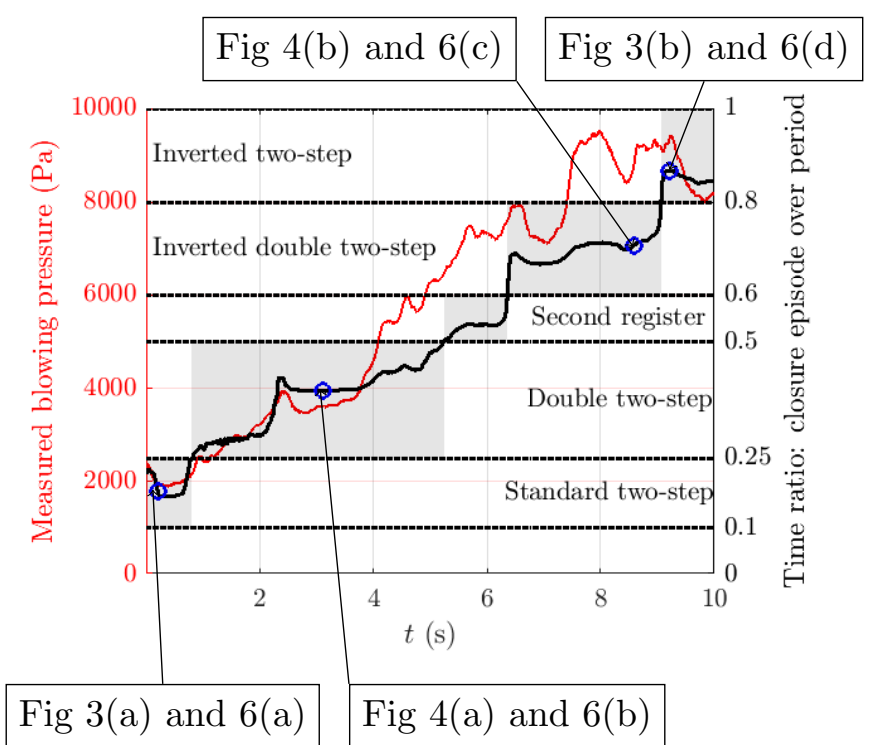

FIG. 5. Result of a blowing pressure increase (low B fingering, alto saxophone) recorded with the instrumented mouthpiece. Left y-axis (red): measured smoothed blowing pressure in Pa. Right y-axis: ratio between closure episode duration and oscillation period (solid black line), and regime separation thresholds (dotted black lines). Greyed areas emphasize the duration of each type of regime. Circles correspond to reed displacement signals in figures 3, 4 and pressure signals in figure 6. 


$$
p=\frac{\hat{p}}{p_{M}} \quad, \quad u=Z_{c} \frac{\hat{u}}{p_{M}} \quad, \quad x=\frac{\hat{x}}{H}
$$

where $p_{M}$ is the static pressure necessary to close the reed completely, $Z_{c}$ is the characteristic impedance at the input of the resonator, and $H$ is the distance separating the reed from the mouthpiece lay at rest. Note that $x=0$ denotes the reed at equilibrium, and $x=-1$ corresponds to a closed reed channel.

The resonator is represented by its dimensionless input impedance, decomposed as a sum of modes

$$
Z(\omega)=\frac{P(\omega)}{U(\omega)}=\sum_{n=0}^{N_{m}} \frac{C_{n}}{i \omega-s_{n}}+\frac{\bar{C}_{n}}{i \omega-\bar{s}_{n}}
$$

where $C_{n}$ are the complex residues and $s_{n}$ the complex poles. These modal parameters are estimated from a measured saxophone input impedance (Taillard et al., 2018). Eq. (2) can be transformed into the temporal evolution of the modal components $p_{n}$, since $j \omega$ translates into a time-domain derivative by inverse Fourier transform

$$
\dot{p}_{n}(t)=s_{n} p_{n}(t)+C_{n} u(t) .
$$


The acoustic pressure $p$ at the input of the tube is expressed as a sum including the modal components

$$
p(t)=2 \sum_{n=1}^{N_{m}} \operatorname{Re}\left(p_{n}(t)\right)
$$

The number of modes $N_{m}$ is chosen as $N_{m}=12$, sufficiently large to represent the main resonances of the resonator. Results obtained using $N_{m}=6$ lead to similar conclusions. The flow $u$ at the input of the resonator is governed by the nonlinear characteristic (Wilson and Beavers, 1974)

$$
u=\zeta[x+1]^{+} \operatorname{sign}(\gamma-p) \sqrt{|\gamma-p|},
$$

where $[x+1]^{+}=\max (x+1,0)$. This nonlinear characteristic uses the dimensionless control parameters of reed opening at rest $\zeta$ and blowing pressure $\gamma$. The expression of these parameters are

$$
\zeta=w H Z_{c} \sqrt{\frac{2}{\rho p_{M}}}, \quad \gamma=\frac{\hat{\gamma}}{p_{M}}
$$

where $w$ is the effective width of the reed channel, $\rho$ the density of air and $\hat{\gamma}$ is the physical value of the blowing pressure. For this study the parameter $\zeta$ is fixed at $\zeta=0.6$, unless otherwise specified. Following the values of reed channel height at rest $H=17 \times 10^{-5} \mathrm{~m}$ and reed stiffness $K=6.4 \times 10^{6}$ Pa.m provided in (Muñoz Arancón et al., 2016), with an approximate effective width of $w=1.10^{-2} \mathrm{~m}$ and characteristic impedance $Z_{c}=$ $3.10^{6}$ Pa.s $/ \mathrm{m}^{3}$, one finds $\zeta=Z_{c} w \sqrt{2 H / \rho K}=0.58$ which justifies studying $\zeta \simeq 0.6 \mathrm{in}$ this work. To use Harmonic Balance Method and Asymptotic Numerical Method, described in subsection III B, it is convenient to regularize the characteristic of Eq. (5) using $|\cdot| \simeq$ $\sqrt{{ }^{2}+\eta}$, where the parameter $\eta$ is fixed at $10^{-3}$ (Kergomard et al., 2016). 
The reed is modeled as a single degree of freedom oscillator driven by the pressure difference between the input of the resonator and the mouth of the resonator

$$
\frac{\ddot{x}}{\omega_{r}^{2}}+q_{r} \frac{\dot{x}}{\omega_{r}}+x=-(\gamma-p),
$$

where $\omega_{r}$ and $q_{r}$ are the angular frequency and damping coefficient of the reed, chosen at $\omega_{r}=4224 \mathrm{rad} / \mathrm{s}$ based on (Muñoz Arancón et al., 2016) and $q_{r}=1$. In this model, the impact of the reed on the mouthpiece lay is ignored(Dalmont et al., 2000; Doc et al., 2014). For further details on the effect of ignoring reed impact in a saxophone model, see (Colinot et al., 2019).

\section{B. Numerical resolution with harmonic balance method}

Periodic solutions to the system of equations (2), (5) and (7) are found using the harmonic balance method (HBM), under the formalism proposed in (Cochelin and Vergez, 2009). The HBM was pioneered by (Krylov and Bogoliubov, 1949; Nakhla and Vlach, 1976), and was applied to musical instrument models first in (Gilbert et al., 1989). Each variable $X$ (where $X$ can stand for $\left.p_{n}, u, x \ldots\right)$ is assumed to be periodic and thus decomposed into its Fourier series truncated at order $H$

$$
X(t)=\sum_{k=-\infty}^{\infty} X_{k} \exp \left(i k \omega_{0} t\right) \simeq \sum_{k=-H}^{H} X_{k} \exp \left(i k \omega_{0} t\right),
$$

where $\omega_{0}$ is the angular frequency. This yield an algebraic system where the unknowns are the Fourier coefficients and the angular frequency. Hereafter, $H=20$ is chosen, because it appears sufficient for a good representation of the studied regimes. The emergence of these different regimes depends on the value of the blowing pressure parameter $\gamma$. To 
compare the value of $\gamma$ leading to each regime to the experimental results of figure 5, a Taylor-series based continuation method (Asymptotic Numerical Method) is applied to the algebraic system obtained by harmonic balance (Guillot et al., 2019). The source code for this method may be found online at http://manlab.lma.cnrs-mrs.fr/. The continuation yields possible periodic solutions, as well as their stability (Bentvelsen and Lazarus, 2018; Lazarus and Thomas, 2010). This may be displayed as a bifurcation diagram representing the evolution of one descriptor of the periodic solutions as a function of the blowing pressure. The bifurcation diagrams displayed here do not change when adding more harmonics, but their computation is more time consuming.

\section{Results}

Depending on the value of the blowing pressure parameter $\gamma$, all types of two-step regimes observed experimentally are found to be stable periodic solutions of the model. Figure 6 compares the regime types found in measurement and simulation from their pressure waveforms. No a posteriori adjustment of the model is performed, and therefore no precise agreement of the waveforms is expected. Many differences between synthesized and measured signals could be explained by the reed opening parameter $\zeta$ being constant and not adjusted in the model, and the response of the pressure probe tube affecting the measured pressure signal. Some high frequency components of synthesized signal can also be misrepresented due to the modal truncation of the impedance. However, several main features of the measured signals can be identified on the synthesized signals, such as the duration of the short low-pressure episodes on the standard and double two-step regimes, and the short 
high-pressure episodes on the inverted double and inverted two-step regimes. It can also be noted that both synthesized and measured signals exhibit secondary fast oscillations of small amplitude during the long episodes (open or closed). A similar "minor oscillations" phenomenon is known to appear on bowed strings (Kohut and Mathews, 1971). The opening duration of the synthesized inverted two-step regime presented in figure $6(\mathrm{~g})$ is longer than the closure duration of the synthesized standard two-step of figure 6 (a), which is contrary to the usual Helmholtz motion formulation in which both durations are determined only by the geometry of the resonator. This is always the case with the model of this paper, with both time-domain synthesis and the harmonic balance: the synthesized and standard and inverted two-step display a whole range of opening or closure durations depending on the value of the blowing pressure. This phenomenon is further detailed below, in multimedia file 5, figure 7 and the corresponding commentary.

The bifurcation diagram summarizing the evolution of the different oscillating regimes depending on the blowing pressure parameter $\gamma$ is presented in figure 7 . A parameter of the oscillating regimes, the amplitude of the first cosine - i.e., the real part of the first Fourier coefficient of Eq. (8) - of the first modal pressure $p_{1}$ is displayed. This parameter was chosen because it allows for clear separation of the branches corresponding to each regime. Note that the sign of this coefficient can be either positive or negative depending solely on a choice of phase of the oscillation. On the diagrams displayed hereafter, the sign of $p_{1}$ chosen so that the different solution branches are as easy to distinguish as possible. The most important part of the branches are stable regimes (thick lines in the figure). Each branch is labeled with the type of regime it corresponds to. The regime type is determined manually by observing the 



FIG. 6. Synthesized and measured pressure signals in the mouthpiece for two-step regimes. Arbitrary units.

waveform, which can be done exhaustively using animations such as multimedia file 5 . Note that the animation shows the standard two-step regime morphing gradually into the inverted two-step regime, on the same branch. The closure duration of the reed increases progressively 
with the blowing pressure parameter $\gamma$, in clear contradiction with the Helmholtz motion approximation. The topic of continuous transition between standard and inverted regimes for a conical woodwind remains to be fully understood, although experimental explorations point to similar results (Dalmont, 2007). All the other branches correspond to only one type of regime each.

Mm. 5. Animation: evolution of the acoustic pressure waveform and spectrum following the stable branches of the bifurcation diagram in figure 7 .

Figure 7 is qualitatively coherent with the experimental findings in figure 5 , in terms of order of emergence of the stable regimes when varying the blowing pressure. Starting with a low blowing pressure, the first stable regime is the standard two-step. When the blowing pressure increases, the stable branch is followed until its end, and then the system jumps on another stable branch. At the end of the standard two-step branch, around $\gamma=0.69$, there are two coexisting branches: the inverted two-step and the double two-step. Note that for the parameter values where two stable regimes coexist, different initial conditions may lead to one or the other. Describing the conditions leading to one or the other regime (called their "attraction basin") exhaustively is almost impossible. Consequently, when using the bifurcation diagram to predict which regimes can be produced when increasing the blowing pressure, several scenarios can be devised, and it is extremely difficult to decide which one is the most probable without checking it experimentally. For instance, according to this bifurcation diagram, it would be possible for the system to start from the standard two-step, jump to an inverted two-step regime and follow this branch until extinction at 
high blowing pressure $(\gamma \simeq 1.5)$, with no production of double two-step regimes. However, we could not obtain this scenario experimentally. Another possible order suggested by the bifurcation diagram, after the standard two-step, is jumping to double two-step, second register, inverted double two-step, and then inverted two-step, when it is the only stable branch (for $\gamma>1.5$ ). The experiment shows that it is possible to obtain all these regimes in this order of emergence when increasing the blowing pressure.

Figure 7 shows that the double two-step branches are linked to the second register branch: a continuum of solutions exist between second register and double two-step motion - even though some of the solutions on the path are unstable. The junction between these branches can be seen as a period-doubling of the second register. Inverted regimes appear at high blowing pressure, which is coherent with the static behavior as the reed tends to close more and more when the blowing pressure is higher. During the oscillation, the reed closes for a longer and longer portion of the period, thus transitioning from standard to inverted motion. A high blowing pressure leads to extinction of the oscillation: the reed channel stays closed. Figure 7 (b) shows the same metric as figure 5, the duration ratio between closure episode and period. It can be noted that the thresholds between the different regimes are not the same as those fixed empirically. Additionnaly, the model predicts that inverted two-step can appear at relatively low closure ratios, but these were never found experimentally. This may be due to the inverted double two-step being very stable in this blowing pressure regions, thus making it hard to find other solutions.

It is worth noting that the same oscillating regimes appear in the same order for other values of the reed opening parameter $\zeta$, around the one used in figure $7(\zeta=0.6)$. Figure 8 


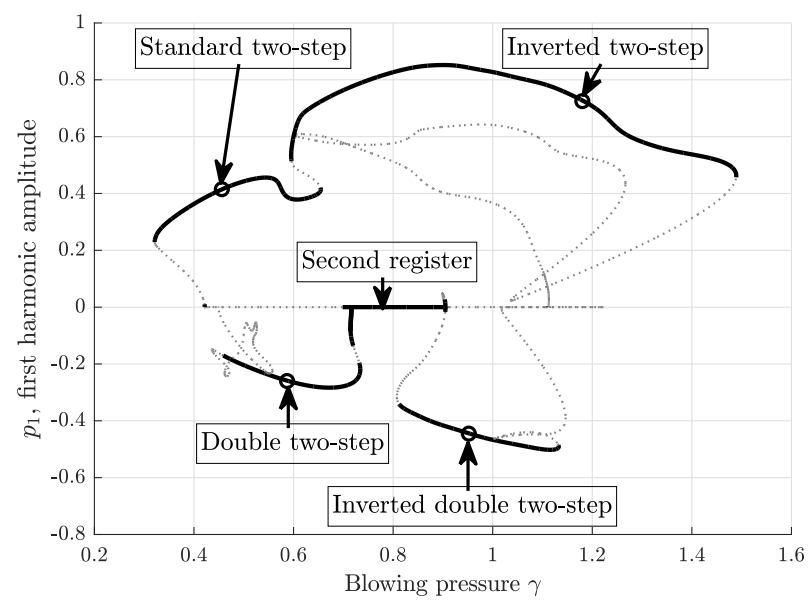

(a)

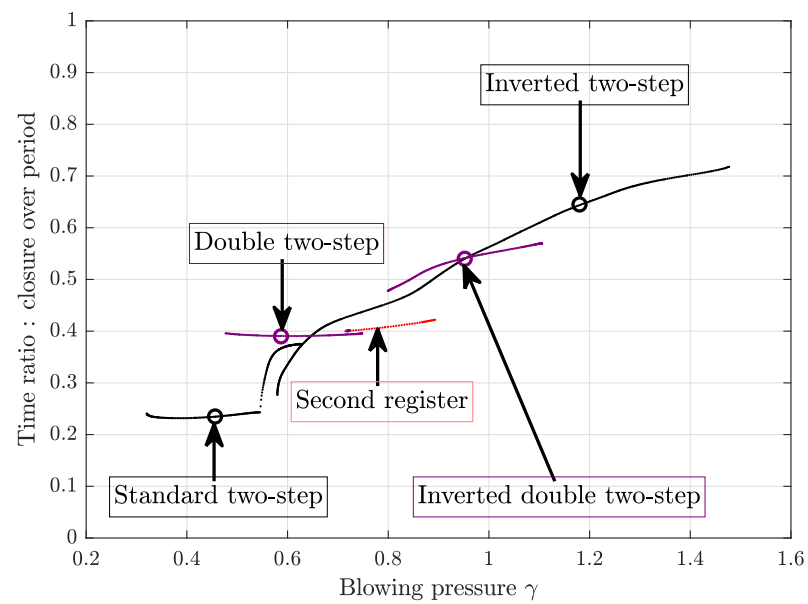

(b)

FIG. 7. Bifurcation diagram: (a) amplitude of the first cosine of the first modal pressure $p_{1}$ and

(b) ratio between closure episode duration and oscillation period ; with respect to the blowing pressure parameter $\gamma$, for the low $B$ fingering of an alto saxophone. In (a), the line aspect denotes stability of the regimes: thick black is stable, dotted gray is unstable. Circle markers correspond to the plots in figure $6 . \zeta=0.6$.

shows two bifurcation diagrams, obtained for $\zeta=0.5$ and $\zeta=0.75$ respectively. The stability region of the regimes are affected by the value of $\zeta$. In particular, a lower $\zeta$ enlarges the zone of stability of the second register while a greater $\zeta$ reduces it. It can also be noted that in this particular case, a higher $\zeta$ value leads to a uninterrupted single two-step branch, where standard and inverted two-step are connected by stable regimes. Another comment can be made on the bifurcation diagram obtained for $\zeta=0.5$ (Figure $8(\mathrm{a})$ ), on the inverted double two-step branch. In this case, the inverted double-two-step branch that is connected to the second register branch only contains unstable regimes - on figure 8 (a) it is the small branch of negative $p_{1}$, between $\gamma=0.86$ and $\gamma=1.04$. This branch corresponds to the branch in figure 7 where the inverted double two-step becomes stable. However, on figure 8 


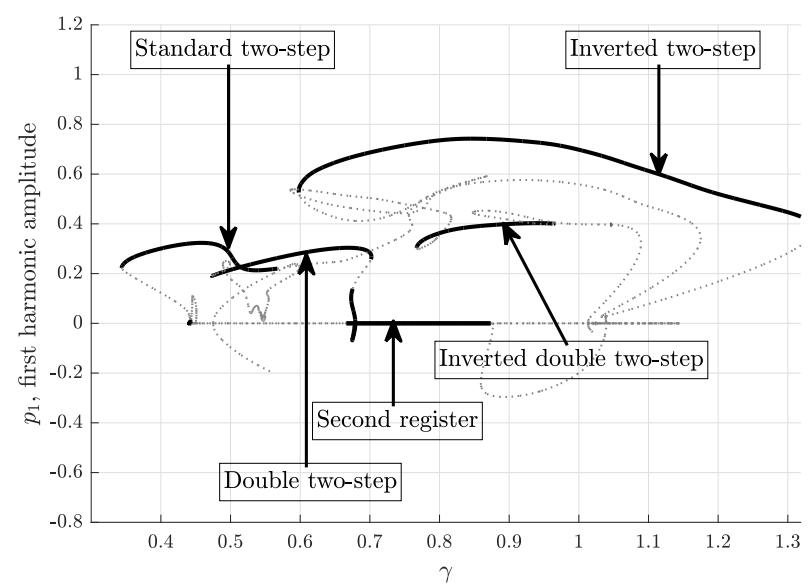

(a)



(b)

FIG. 8. Bifurcation diagram: amplitude of the first cosine of the first modal pressure $p_{1}$ with respect to the blowing pressure parameter $\gamma$, for the low $B$ fingering of an alto saxophone. (a) $\zeta=0.5$, (b) $\zeta=0.75$. The line aspect denotes stability of the regimes: thick black is stable, dotted gray is unstable

(a), another inverted double two-step branch shows stable regimes, that are indicated by the inverted double two-step arrow. This other branch is not connected to the second register, but to the inverted single two-step branch, by a long unstable portion of branch. Therefore it appears that double two-step regimes can be considered as degenerate from the single two-step or the second register, depending on the value of the control parameters. $\zeta=0.5,(b) \zeta=0.75$. The line aspect denotes stability of the regimes: thick black is stable, dotted

A similar behavior is also observed for neighboring fingerings. Figure 9 shows the bifurcation diagram for the fingering just above the one used for figures 7 and 8: the low $C$ fingering. The bifurcation diagram in figure 9 has the same structure as the others, although the inverted double two-step regime is unstable. In particular, the transition between standard two-step and inverted two-step regimes is an unstable portion of branch featuring two fold bifurcations (two points where two solutions collide and disappear, which can be 
seen as turning-up points on the bifurcation diagram), similar to that of figure 8 , up, and figure 7. It is also worth noting that on this fingering, the double two-step branch and second register branch are connected by stable regimes only: the thick lines connect at $\gamma=0.8$. This indicates that for this fingering, it is possible to have continuous transition between double two-step and second register using only stable regimes. A synthesized example of this transition is shown in multimedia file 6 .

Mm. 6. Animation: evolution of the acoustic pressure waveform and spectrum during a continuous transition between double two-step regime and second register for the low $C$ fingering of an alto saxophone, following branches of the bifurcation diagram in figure 9 .

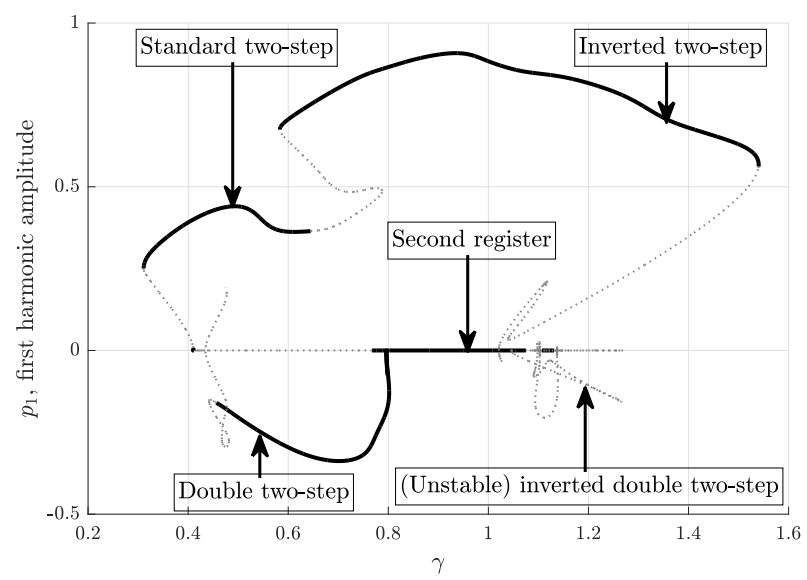

FIG. 9. Bifurcation diagram: amplitude of the first cosine of the first modal pressure $p_{1}$ with respect to the blowing pressure parameter $\gamma$, for the low $C$ fingering of an alto saxophone. $\zeta=0.6$, same as in figure 7 .

The double two-step regime becomes unstable on fingerings D and higher for the main value of $\zeta=0.6$ studied here. This may be a sign that its production is linked to the high 
amplitude of the second and third resonances of the resonator, which is a characteristic of the low fingerings of the saxophone.

\section{CONCLUSION}

Alto saxophones are able to produce double two-steps motions, that seem analogous to double stick-slip motions in bowed strings (Woodhouse, 2014). The production region of these regimes appears linked to the second register of the resonator. The appearance of the many oscillating regimes on the studied fingerings may be due to the strong role of the second and third mode of the resonator. The simple saxophone model used in this paper is capable of reproducing these regimes, even though it ignores the impact between the reed and the mouthpiece lay. The model also corroborates the order of appearance of these regimes when increasing the blowing pressure on a real saxophone. Complementary numerical studies show that the double two-step phenomenon is not restricted to a particular set of parameters, but appears for several combinations of control parameters and several fingerings. The description of the playability of a saxophone in the low fingerings may take these regimes into account, whether they are undesirable, as is the case for the double fly-back motion in violins, or a useful tool of expressivity for the musician. Acoustical or geometrical characteristics of the resonator remain to be linked to the ease of production of double two-step regimes. 


\section{ACKNOWLEDGMENTS}

The authors would like to thank Louis Guillot for advice and guidance in the construction of the bifurcation diagram. This work has been carried out in the framework of the Labex MEC (ANR-10-LABX-0092) and of the A*MIDEX project (ANR-11-IDEX-000102), funded by the Investissements d'Avenir French Government program managed by the French National Research Agency (ANR). This study has been supported by the French ANR 659 LabCom LIAMFI (ANR-16-LCV2-007-01).

Bentvelsen, B., and Lazarus, A. (2018). "Modal and stability analysis of structures in periodic elastic states: application to the ziegler column," Nonlinear Dynamics 91(2), 1349-1370.

Chen, J.-M., Smith, J., and Wolfe, J. (2009). "Saxophone acoustics: introducing a compendium of impedance and sound spectra," Acoustics Australia 37(1-19).

Cochelin, B., and Vergez, C. (2009). "A high order purely frequency-based harmonic balance formulation for continuation of periodic solutions," Journal of sound and vibration 324(12), 243-262.

Colinot, T., Guillot, L., Vergez, C., Guillemain, P., Doc, J.-B., and Cochelin, B. (2019). "Influence of the "ghost reed" simplification on the bifurcation diagram of a saxophone model," Acta Acustica united with Acustica [In press](-), -. 
Dalmont, J.-P. (2007). "Analytical and experimental investigation of the dynamic range of conical reed instruments," in Proceedings of the International Symposium on Musical Acoustics, Barcelona, Spain.

Dalmont, J.-P., Gilbert, J., and Kergomard, J. (2000). "Reed instruments, from small to large amplitude periodic oscillations and the Helmholtz motion analogy," Acta Acustica united with Acustica 86(4), 671-684.

Dalmont, J.-P., and Le Roux, J. C. (2008). "A new impedance sensor for wind instruments," The journal of the Acoustical Society of America 123(5), 3014-3014.

Dalmont, J.-P., and Le Vey, G. (2014). "The irish uillean pipe: a story of lore, hell and hard d," in International Symposium on Musical Acoustics.

Doc, J.-B., Vergez, C., and Missoum, S. (2014). "A minimal model of a single-reed instrument producing quasi-periodic sounds," Acta Acustica united with Acustica 100(3), $543-554$.

Gilbert, J., Kergomard, J., and Ngoya, E. (1989). "Calculation of the steady-state oscillations of a clarinet using the harmonic balance technique," The journal of the Acoustical Society of America 86(1), 35-41.

Guillemain, P., Vergez, C., Ferrand, D., and Farcy, A. (2010). "An instrumented saxophone mouthpiece and its use to understand how an experienced musician plays," Acta Acustica united with Acustica 96(4), 622-634.

Guillot, L., Cochelin, B., and Vergez, C. (2019). "A taylor series-based continuation method for solutions of dynamical systems," Nonlinear Dynamics 1-19. 
Keefe, D. H. (1984). “Acoustical wave propagation in cylindrical ducts: Transmission line parameter approximations for isothermal and nonisothermal boundary conditions," The Journal of the Acoustical Society of America 75(1), 58-62.

Kergomard, J., Guillemain, P., Silva, F., and Karkar, S. (2016). "Idealized digital models for conical reed instruments, with focus on the internal pressure waveform," The Journal of the Acoustical Society of America 139(2), 927-937.

Kohut, J., and Mathews, M. (1971). "Study of motion of a bowed violin string," The Journal of the Acoustical Society of America 49(2B), 532-537.

Krylov, N. M., and Bogoliubov, N. N. (1949). Introduction to non-linear mechanics (Princeton University Press).

Lazarus, A., and Thomas, O. (2010). "A harmonic-based method for computing the stability of periodic solutions of dynamical systems," Comptes Rendus Mécanique 338(9), 510-517. Muñoz Arancón, A., Gazengel, B., Dalmont, J.-P., and Conan, E. (2016). "Estimation of saxophone reed parameters during playing," The Journal of the Acoustical Society of America 139(5), 2754-2765.

Nakhla, M., and Vlach, J. (1976). "A piecewise harmonic balance technique for determination of periodic response of nonlinear systems," IEEE Transactions on Circuits and Systems 23(2), 85-91.

Ollivier, S., Dalmont, J.-P., and Kergomard, J. (2004). "Idealized models of reed woodwinds. Part I: Analogy with the bowed string," Acta acustica united with acustica 90(6), 11921203. 
Ollivier, S., Kergomard, J., and Dalmont, J.-P. (2005). "Idealized models of reed woodwinds. Part II: On the stability of "two-step" oscillations," Acta acustica united with acustica 91(1), 166-179.

Pàmies-Vilà, M., Hofmann, A., and Chatziioannou, V. (2018). "Analysis of tonguing and blowing actions during clarinet performance," Frontiers in psychology 9.

Schelleng, J. C. (1973). "The bowed string and the player," The Journal of the Acoustical Society of America 53(1), 26-41.

Taillard, P.-A., Silva, F., Guillemain, P., and Kergomard, J. (2018). "Modal analysis of the input impedance of wind instruments. application to the sound synthesis of a clarinet," Applied Acoustics 141, 271-280.

Wilson, T. A., and Beavers, G. S. (1974). "Operating modes of the clarinet," The Journal of the Acoustical Society of America 56(2), 653-658.

Woodhouse, J. (2014). "The acoustics of the violin: a review," Reports on Progress in Physics 77(11), 115901. 